2004;26:213-218). Most of the known genes code for ion channels, but 2 are not channelopathies (MASS1/VLGR1 coding for a G-protein coupled receptor in one family with febrile and afebrile seizures, and LG11, a tumor suppressor gene, identified in several families with autosomal dominant (AD) familial lateral temporal lobe epilepsy) Other epilepsies with identified genes are AD familial nocturnal frontal lobe epilepsy (CHRNA4), generalized epilepsy with febrile seizures plus (SCN1A, SCN2A), severe myoclonic epilepsy of infancy (SCN1A), and juvenile myoclonic epilepsy (GABRA1). A large number of genetic factors probably contribute to seizure susceptibility.

The clinical spectrum of SCN1A mutations ranges from febrile seizures, febrile seizures plus, a mild and a classical form of severe myoclonic epilepsy in infancy (Dravet syndrome). (Berten PGM et al. Clinical correlations of mutations in the SCN1A gene: from febrile seizures to severe myoclonic epilepsy in infancy. Pediatr Neurol April 2004;30:236243).

\title{
EPILEPSY RISK FACTORS FOLLOWING NEONATAL SEIZURES
}

Clinical and polysomnographic risk factors as early predictors for the development of postnatal epilepsy were determined in 158 infants presenting with two or more seizures, in a study at Hospital Sao Lucas, Porto Alegre, Brazil. Epilepsy rate after neonatal seizures was $22 \%$ within 12 months follow-up and $33.8 \%$ within 48 months. The types of epilepsy syndromes included: West syndrome in 12 infants; focal symptomatic epilepsy in 10; infantile epileptic encephalopathy in 3; other generalized symptomatic epilepsies in 5; and miscellaneous (13). Perinatal asphyxia, electrolyte imbalance, and bacterial meningitis were the most frequent etiologic factors in neonatal seizures. An abnormal neurologic examination, cerebral palsy, cognitive deficits, and abnormal polysomnographic recording were predictors of an unfavorable outcome and development of postnatal epilepsy. Prematurity, birth weight, and perinatal asphyxia were not significantly related to outcome, but the incidence of postnatal epilepsy was significantly correlated with neonatal bacterial meningitis. Neonates with seizures that required large initial doses and maintenance anticonvulsant drugs were at greater risk of postnatal epilepsy. (Da Silva LFG, Nunes ML, Da Costa JC. Risk factors for developing epilepsy after neonatal seizures. Pediatr Neurol April 2004;30:271-277). (Respond: Dr Nunes, Division of Neurology, Hospital Sao Lucas, Pontificia Universidade Catolica do Rio Grande do Sul School of Medicine, Av Ipiranga 6690, cj220, 90610-000 Porto, Alegre, RS, Brazil).

COMMENT. In this selected population that included infants in intensive care, a higher incidence of epilepsy than that seen in population-based studies could be expected. Multiple risk factors for neonatal seizures, and especially bacterial meningitis, are associated with a higher probability of developing postnatal epilepsy. An abnormal neurologic examination and polysomnographic abnormalities are predictors of an unfavorable outcome.

\section{HYPOTHYROIDISM AND NEONATAL SEIZURES}

A term infant born to a mother with gestational diabetes and a history of hypothyroidism presented with seizures on the $6^{\text {th }}$ day of life that responded to L-thyroxine, in a report from The Children's National Medical Center, Washington, DC. The mother had 